\title{
Infrequent disposition of the first metacarpal artery related to anastomoses of the superficial and deep systems of the hand
}

\author{
H.F. Bianchi ${ }^{1,2}$, N.E. Ottone ${ }^{3}$ (1) \\ ${ }^{1}$ Anatomy Department, Medicine School, University of Buenos Aires, Argentina \\ ${ }^{2}$ Anatomy Department, Instituto Universitario de Ciencias de la Salud, Fundacion Barcelo, Buenos Aires, Argentina \\ ${ }^{3}$ Laboratory of Plastination and Anatomical Techniques, Research Centre for Dental Sciences (CICO), Department of \\ Adults Integral Odontology, Dental School, Universidad de La Frontera, Temuco, Chile
}

[Received: 2 December 2019; Accepted: 30 March 2020]

\begin{abstract}
There is an infrequent disposition of the first metacarpal artery which results in an anastomosis with the common palmar digital artery of the second space, forming an anastomotic system between the deep radial system and the superficial ulnar system. The radial system in turn, through the first dorsal interosseous artery, anastomoses with a collateral of the common palmar digital artery of the second space, ending as the ulnar palmar digital artery of the thumb and establishing a second anastomotic system. A third anastomosis is established in one case, between the common palmar digital artery of the fourth space and the fourth metacarpal artery. To this may be added the classic anastomosis between the superficial branch of the ulnar artery and the deep branch of the radial artery, i.e. the deep palmar arch. (Folia Morphol 2021; 80, 2: 448-454)
\end{abstract}

Key words: first metacarpal artery, superficial palmar arch, deep palmar arch, anastomosis

\section{INTRODUCTION}

In the course of an investigation into the morphology of the superficial palmar arch (SPA), we found an infrequent disposition of the first palmar metacarpal artery (1PMA), which is considered primordial in the circulation of the thumb to the point that it is called the princeps pollicis artery. We believe that surgeons must be familiar with the arterial disposition of this finger for the purposes of surgery and reconstruction procedures.

\section{MATERIALS AND METHODS}

The specimens were 28 fresh upper limbs undistinguished by sex, mean age 65 years. The specimens were injected manually, through axillary artery, with elastomeric material (natural latex) (Meister $\left.{ }^{\circledR}\right)$, using $10 \mathrm{~mL}$ syringes. Chemically, this elastomeric material is defined as a polymer of methylbutadiene or isoprene $\left(\mathrm{C}_{5} \mathrm{H}_{8}\right)$, whose degree of polymerisation is 2500 to 4500 . It is white in colour, and was coloured red with an acrylic paint (chemically, vinyl acetate) (Arcel ${ }^{\odot}$ ). Subsequently, the specimens were immersed in a $10 \%$ formalin fixative liquid container for 30 days, at room temperature. The upper limbs were dissected using appropriate instruments and stereoscopic microscopes $2 x$.

\section{RESULTS}

The findings reported were found in 3 cases, i.e. $10.7 \%$, of the total sample.

Address for correspondence: Prof. Dr. N.E. Ottone, MD, PhD, Laboratory of Plastination and Anatomical Techniques, Research Centre for Dental Sciences (CICO), Dental School, Universidad de La Frontera, Francisco Salazar 01145, Casilla 54-D, Temuco, Chile, tel: +56452596935, e-mail: nicolas.ottone@ufrontera.cl

This article is available in open access under Creative Common Attribution-Non-Commercial-No Derivatives 4.0 International (CC BY-NC-ND 4.0) license, allowing to download articles and share them with others as long as they credit the authors and the publisher, but without permission to change them in any way or use them commercially. 
Common characteristics were observed between cases 1 and 2. The SPAs of cases 1 and 2 may be considered of the incomplete type (Figs. 1A, 2A): the 1 PMA originates in the deep palmar arch (DPA), close to the passage of the radial artery (RA) through the first dorsal interosseous muscle (1DIOM) (Figs. 1C, 2C). From this origin, the artery lies between the first dorsal interosseous muscle (1DIOM) and the adductor pollicis (AdP), emerging below its distal border, hidden by the flexor tendons of the index finger. At the point of emergence, it emits the radial artery of the index (Figs. 1A, B; 2A, B), which is seen emerging outside the flexor tendons of this finger. Located at first outside the flexor tendons, the 1PMA passes below them close to the metacarpophalangeal joint, and subsequently courses inside the flexor tendons (Figs. 1A, B; 2A, B) in the second interdigital space, where it anastomoses with the common palmar digital artery (CPDA) of the second space (CPDA2S) (Figs. 1A, B; 2A, B). It courses posterior of the palmar digital nerves of the index finger. Before it passes through the 1DIOM ring, the RA emits the first dorsal interosseous artery (1DIOA) (Figs. 1C, 2C), which anastomoses with a branch of the SPA at the edge of the first commissure. It continues its course parallel to the medial border of the thumb to become the ulnar palmar artery of the thumb (UPAT) (Figs. 1C, 2C). In case 1, shortly after its origin, the CPDA2S emits a branch which courses to proximal and divides into two branches (Fig. 1A): one distal, parallel to the medial border of the thumb, which anastomoses with the 1DIOA when it reaches the border of the first joint; and one proximal which ends up as the radial palmar artery of the thumb. Case 2 is similar (Fig. 2A), but it is the branch that anastomoses with the dorsal system that emits the radial palmar artery of the thumb. In case 1 (Fig. 1A), other arterial variations were observed: the origin of a common trunk for the CPDA of the fifth finger and the common palmar digital artery of the fourth space (CPDA4S), which has to pass below the extensor tendons and the common palmar digital nerve of this space, to arrive at the same point, receiving a fourth palmar metacarpal artery (4PMA) with which it anastomoses; and the presence of a highly developed second dorsal interosseous artery (2DIOA) located over the second dorsal interosseous muscle, which passes under the extensor tendons of the index and the junctura tendinum between this and the middle finger, and ends by joining the CPDA2S at the point where it divides into the medial and radial proper palmar digital artery of the index and middle fingers (Fig. 1D).

Case 3 presents a complete SPA (Fig. 3A), with the thumb receiving blood supply from the ulnar proper palmar digital artery of the thumb and from radial proper palmar digital artery of the thumb, originating in the CPDA of the first space. The RA does not give origin to a 1DIOA. The 1PMA originates from the DPA and presents the same course and disposition as in the above cases (Fig. 3B, C). It also presents an arterial variation in the form of a DPA between the RA and a distal deep ulnar artery (Fig. 2C). The proximal deep ulnar artery turns over the DPA and a small anastomosis can be seen between the first and second metacarpal arteries (Fig. 3C).

\section{DISCUSSION}

Although our purpose is not to discuss the origins of the arteries of the thumb, in order to compare our findings we will say that the 1PMA has been considered the principal source of irrigation of the thumb, which is why it has been called princeps pollicis [15, 21]. Other authors argue that it should not be so called, as its contribution to the vascularisation of the thumb is inconstant $[10,15,16,18]$, or because its contribution is provided from other sources: 1DIOA $[1-3,9,13,15,16,18]$; or SPA $[1,11,15,17]$. Other authors again consider that the 1PMA and the princeps pollicis are different $[8,19]$. A similar disposition to our findings appears in illustration by Dujarier [9], who calls the 1PMA as the arterial trunk of the index and - citing Farabeuf - considers this a habitual disposition, an opinion shared by Delorme [8]. Patil et al. [13] refers to a case, which we understand to be the same as those described here, in which a 1PMA is located in the second palmar space with termination as in our findings, but with the RA of the index passing under the tendons and nerves destined for the index, because the 1PMA is located in a medial position relative to these structures, while in our specimens it passes to radial. A similar disposition to ours is mentioned by Hashem et al. [12], without indicating which metacarpal artery is substituted. Occasionally a second palmar metacarpal artery (2PMA) can supply a CPDA of the second space [2], forming another anastomosis between elements of the DPA and the SPA [3, 4]. Coleman and Anson [7] say that the most developed artery destined for thumb should be called 1PMA.

Our cases confirm that it is not correct to call 1 PMA as "princeps pollicis", because it does not con- 

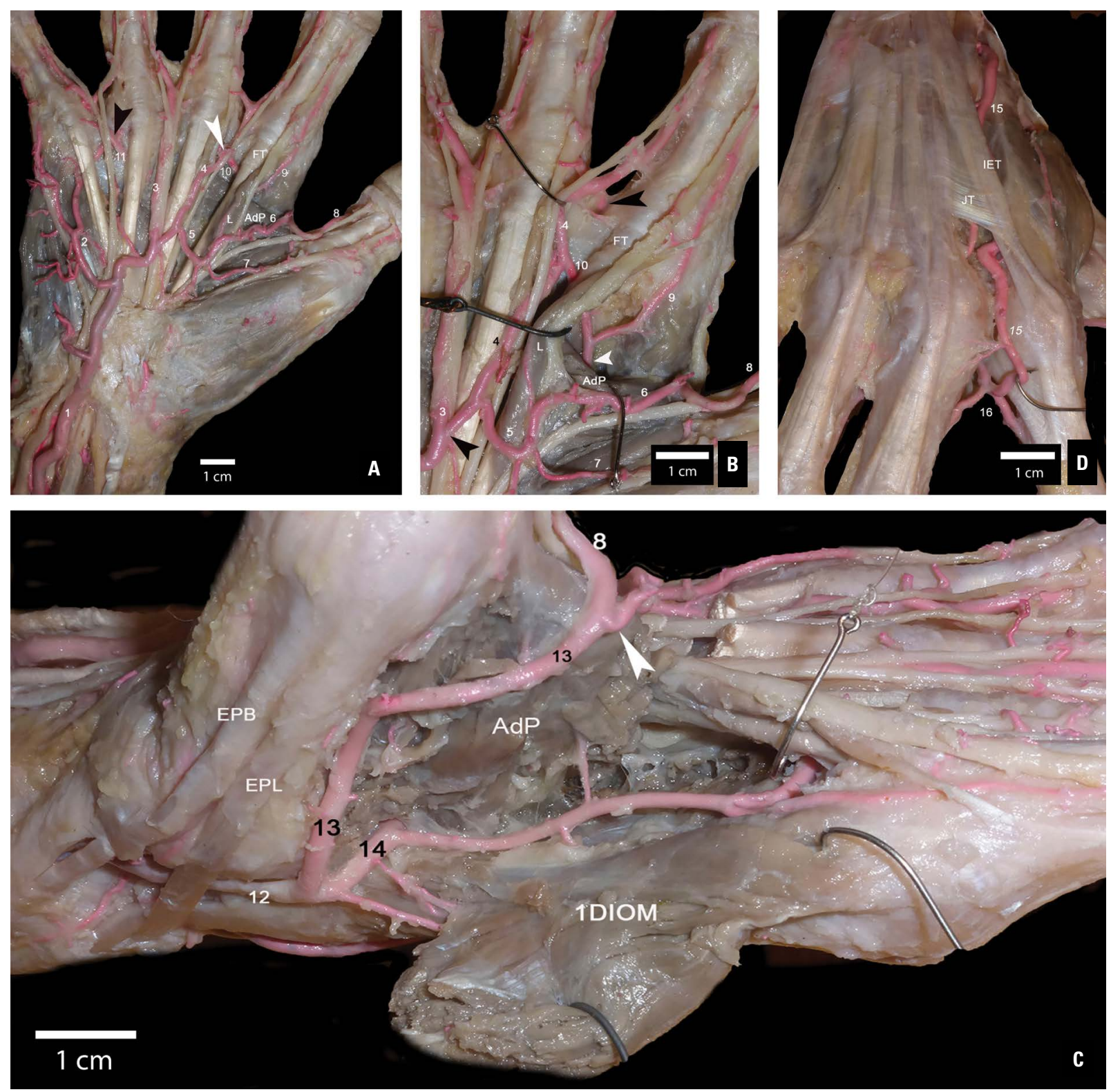

Figure 1. A. View of the palmar side of the hand; 1 - ulnar artery; 2 - common trunk for the common palmar digital artery of the fifth finger and the common palmar digital artery of the fourth space; 3 - common palmar digital artery of the third space; 4 - common palmar digital artery of the second space; 5 - branch of the common palmar digital artery of the second space; 6 - distal anastomotic branch of the first dorsal interosseous artery; 7 - proximal branch of the first dorsal interosseous artery; 8 - ulnar proper palmar digital artery of the index; 9 - radial artery of the index; 10 - anastomotic branch between first palmar metacarpal artery and common palmar digital artery of the second space (white arrow); 11 — common palmar digital artery of the fourth space (black arrow); FT — flexor tendons; L — lumbrical muscle; AdP - adductor pollicis muscle; B. Enlarged visualization of palmar side of the hand on the first and second interosseous space; white arrow — first palmar metacarpal artery; black arrow: anastomosis between second dorsal interosseous artery and common palmar digital artery of the second space; 3 - common palmar digital artery of the third space; 4 - common palmar digital artery of the second space; 5 - branch of the common palmar digital artery of the second space; 6 - distal anastomotic branch of the first dorsal interosseous artery; 7 - proximal branch of the first dorsal interosseous artery; 8 - ulnar proper palmar digital artery of the index; 9 - radial artery of the index; 10 - anastomotic branch between first palmar metacarpal artery and common palmar digital artery of the second space (white arrow); FT — flexor tendons; AdP — adductor pollicis muscle; C. View of the dorsal side of the hand; 12 - radial artery; 13 - first dorsal interosseous artery (white arrow: anastomosis with medial branch of common palmar digital artery of the second space); 8 - ulnar proper palmar digital artery of the index; 14 - first palmar metacarpal artery; $1 \mathrm{DIOM}$ - first dorsal interosseous muscle (reclined and sectioned at the level of the interosseous ring); AdP — m. adductor pollicis; EPB — m. extensor pollicis brevis; EPL — m. extensor pollicis longus; D. View of the dorsal side of the hand, from the first interosseous space, with recline of the first dorsal interosseous muscle; 15 - second dorsal interosseous artery; 16 — proper palmar digital arteries; IET — index extensor tendons; JT — juncturae tendineum. 

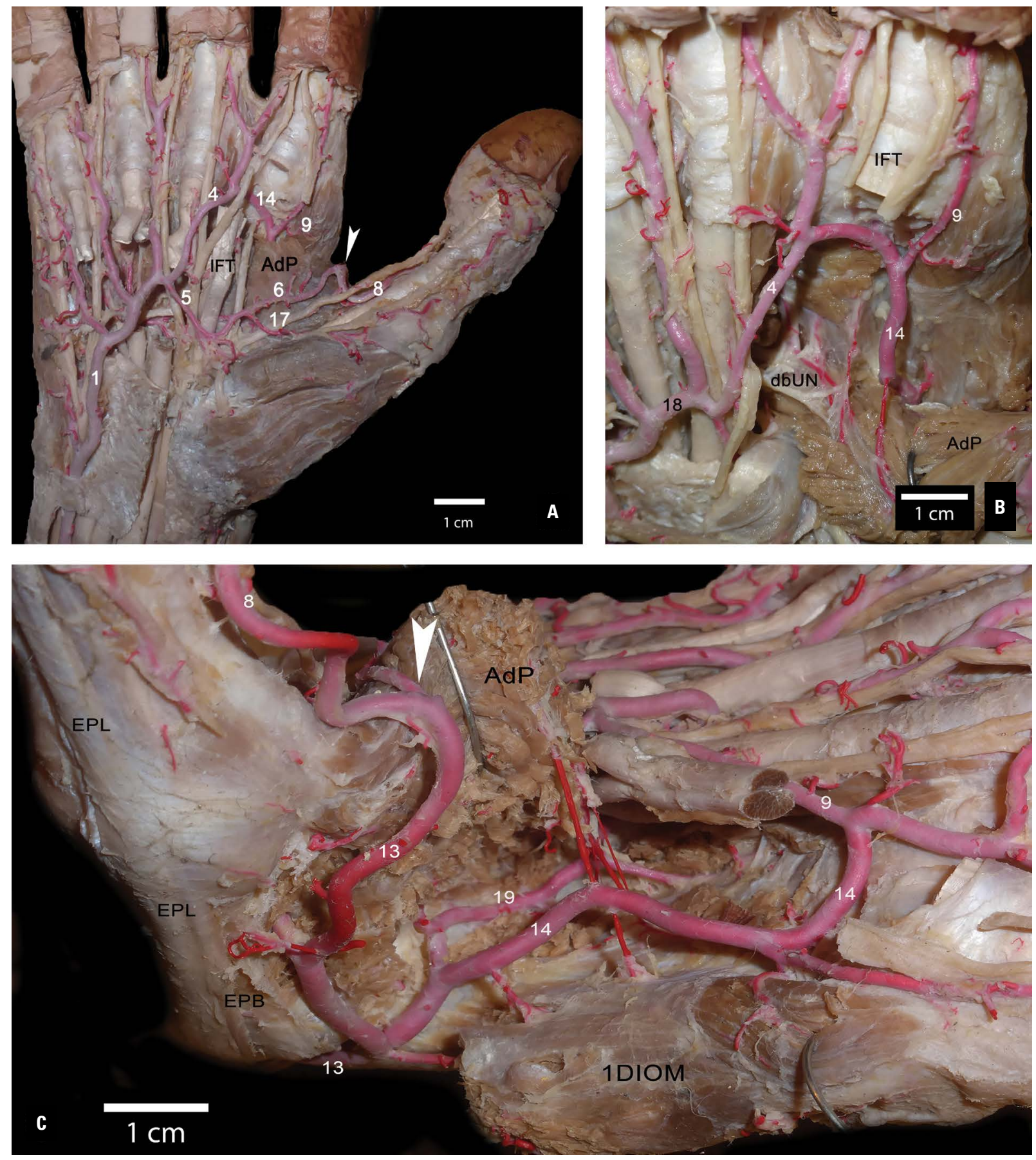

Figure 2. A. View of the palmar side of the hand; 1 - ulnar artery; 4 - common palmar digital artery of the second space; 5 - branch of the common palmar digital artery of the second space; 6 - distal anastomotic branch of the first dorsal interosseous artery; 8 - ulnar proper palmar digital artery of the thumb; 9 - radial artery of the index; 14 - first palmar metacarpal artery; 17 - radial proper palmar digital artery of the thumb; AdP — m. adductor pollicis; IFT — index flexor tendons; B. Enlarged visualization of palmar side of the hand; 4 - common palmar digital artery of the second space; 9 - radial artery of the index; 14 - first palmar metacarpal artery; 18 - superficial palmar arch; AdP — m. adductor pollicis; dbUN — deep branch of the ulnar nerve; IFT — index flexor tendons; C. View of the dorsal and palmar side of the hand, from the first interosseous space, with recline of the first dorsal interosseous muscle; 8 - ulnar proper palmar digital artery of the thumb; 9 - radial artery of the index; 13 - first dorsal interosseous artery; 14 - first palmar metacarpal artery; 19 - deep palmar arch; AdP — m. adductor pollicis; EPB — m. extensor pollicis brevis; EPL — m. extensor pollicis longus; 1DIOM — first dorsal interosseous muscle; white arrow - anastomosis between distal branch of the common palmar digital artery of the second space with the first dorsal interosseous artery. 

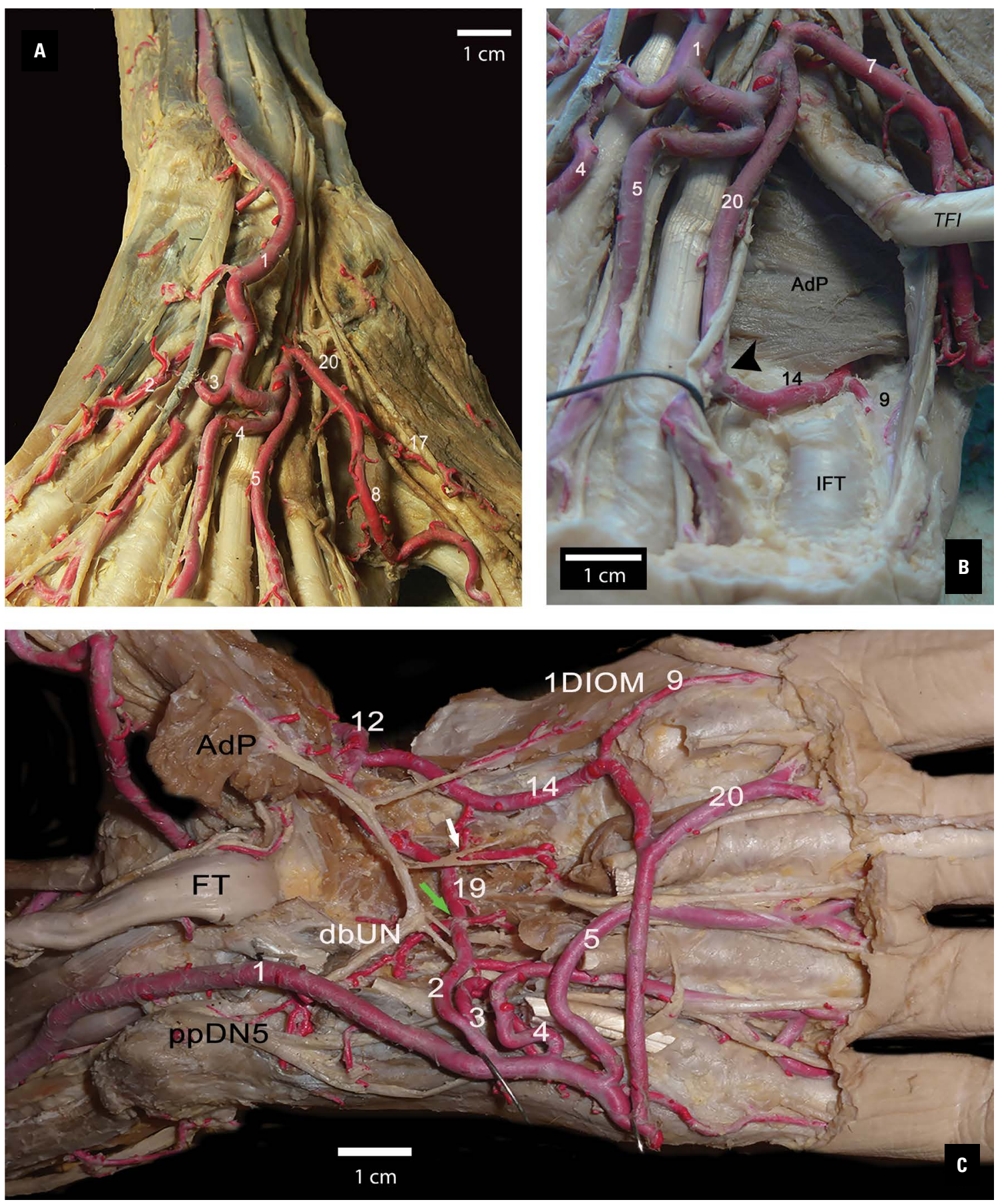

Figure 3. A. View of the palmar side of the hand; 1 - ulnar artery; 2 - common trunk for the common palmar digital artery of the fifth finger and the common palmar digital artery of the fourth space; 3 - common palmar digital artery of the third space; 4 - common palmar digital artery of the second space; 5 - branch of the common palmar digital artery of the second space; 8 - ulnar proper palmar digital artery of the thumb; 17 - radial proper palmar digital artery of the thumb; 20 - common palmar digital artery of the first space; B. Enlarged visualisation of palmar side of the hand, with recline of the index flexor tendon, and visualisation of $\mathrm{m}$. adductor pollicis; 1 - ulnar artery; $4-$ common palmar digital artery of the second space; 5 - branch of the common palmar digital artery of the second space; 7 proximal branch of the first dorsal interosseous artery; 9 - radial artery of the index; 14 - first palmar metacarpal artery; 20 - common palmar digital artery of the first space; AdP — m. adductor pollicis; IFT — index flexor tendons; black arrow — anastomosis with common palmar digital artery of the second space; C. View of the palmar side of the hand, with recline of the thumb; 1 - ulnar artery; 2 - common trunk for the common palmar digital artery of the fifth finger and the common palmar digital artery of the fourth space; 3 - common palmar digital artery of the third space; 4 - common palmar digital artery of the second space; 5 - branch of the common palmar digital artery of the second space; 9 - radial artery of the index; 12 - radial artery; 14 - first palmar metacarpal artery; 19 - deep palmar arch; 20 - common palmar digital artery of the first space; white arrow - anastomosis between first palmar metacarpal artery and second palmar metacarpal artery; green arrow — anastomosis between deep branch of ulnar artery and deep palmar arch; ppDN5 — proper palmar digital nerve of fifth digit; $\mathrm{dbUN}$ — deep branch of the ulnar nerve; FT — flexor tendons; AdP — m. adductor pollicis; 1DIOM — first dorsal interosseous muscle. 
tribute to the vascularisation of the thumb in any of them: in two cases this is provided by 1DIOA which ends up as ulnar proper palmar digital artery of the thumb. In case 1 the radial proper palmar digital artery of the thumb originates from the branch which has originated in the CPDA2S, and in case 2 from the distal branch from the same origin, anastomosing with the dorsal system. In case 3 , both radial proper palmar digital artery of the index come from the SPA.

In all cases, the 1PMA (Fig. 1A) presents an anastomosis which differs from the classic configuration between the deep radial and the superficial ulnar systems via an indirect anastomosis between two collateral branches of those systems, the CPDA2S and the 1PMA, in the second digital space, although it originates in the first space. The same occurs in other cases of anastomosis between the radial and ulnar systems, most frequently originating in the SPA and the 1DIOA $[1,2,15,18]$. Anastomoses between the SPA and the 1PMA or princeps pollicis were mentioned by Browning and Morton [6], but none of these cases are similar to our findings. In all the cases investigated, the 1PMA supplies the radial artery of the index $[15,16,18]$. We do not think it appropriate, even in cases like this, to call it the arterial trunk of the index as Dujarier does [9], although it could be justified in case 2 , since the volume of the 1PMA is greater than that of the CPDA, which appears to be anastomosed with the 1PMA and not the other way round. The volume of the ulnar proper palmar digital artery of the thumb supplied by the 1DIOA is dominant, coinciding with other findings $[15,18]$; this justifies preference for its use in implants and revascularisation [19]. In 2 of our findings, the RA was the principal source of irrigation of the thumb [1].

In specimen 1, simultaneous arterial variations were observed, such as presence of the common trunk of origin of the CPDAs for the fifth and fourth spaces, similar to that reported by Singh et al. [14] and Hasham [12]; however, these authors identified a different course of the common trunk, as well as an anastomosis of the CPDA of the fourth finger with a 4PMA, and a 2DIOA. This anastomosis reinforced the circulation of the second interdigital space instead of supplying it [3], giving rise to another indirect anastomosis between the SPA and DPA.

We agree with Rodríguez-Niedenführ et al. [20], who considered that the arteries develop from shoots, initially capillary plexuses, and that they become differentiated from proximal to distal. In this process, some become definitive blood vessels, while others disappear or present incomplete development.

Although the incidence of modifications in arterial disposition is low, occlusions of the RA due to cannulation or trauma (Mozersky cited by Parks [18]) may have consequences such as ischaemia or acute vascular insufficiency of the thumb. We believe that caution must be exercised in surgical approaches to the radial side of the hand, where arterial variations are more frequent $[4,5]$, for example in aponeurotic retractions, lesions of the flexor tendons of the index, and in primary or secondary repairs. There is also a risk in the pollicisation of the index finger [12]. In some cases, the use of a Doppler ultrasound scan or angiography needs to be considered to ensure that the surgery is well planned.

\section{CONCLUSIONS}

We confirm the presence of anastomotic circuits between the superficial and deep systems of the hand, apart from the classic anastomosis between the SPA and DPA, occurring between their collateral arteries: 1PMA and CPDA2S, 1DIOA and SPA, 4PMA with CDPA4S, 2DIOA and proper palmar digital artery of the second interdigital space, in addition to those reported between the 2PMA and the CPDA. Their presence may explain why many serious hand injuries have a favourable evolution, including injuries to the thumb where the circulation is not restricted to a single artery. Considering the frequency of arterial modifications in the radial side of the hand, their possible presence must be taken in mind when carrying out both invasive and non-invasive procedures.

\section{Ethical considerations}

We complied with all the protocols and requirements established by the Government of the City of Buenos Aires, Argentina ("Protocol for the treatment of dead bodies, dead births, segments and anatomical parts in GCBA hospitals. Government of the City of Buenos Aires, Argentina, 2014").

\section{REFERENCES}

1. Ames EL, Bissonnette $M$, Acland $R$, et al. Arterial anatomy of the thumb. J Hand Surg. 1993; 18(4): 427-436, doi: 10.1016/0266-7681(93)90141-2, indexed in Pubmed: 8409651.

2. Bataineh ZM, Moqattash ST. A complex variation in the superficial palmar arch. Folia Morphol. 2006; 65(4): 406-409, indexed in Pubmed: 17171624.

3. Bianchi HF, Leiro R. The arterial trunk of the thumb-index digital collaterals. Surg Radiol Anat. 1987; 9(1): 63-67, doi: 10.1007/BF02116855, indexed in Pubmed: 3112980. 
4. Bianchi H. Anatomy of the radial branches of the palmar arch. Variations and surgical importance. Hand Clin. 2001; 17(1): 139-146, doi: 10.1016/s0749-0712(21)00607-7, indexed in Pubmed: 11280157.

5. Bianchi HF, Vargas CA, Ottone NE. Unusual ending of the second dorsal metacarpal artery as proper palmar digital arteries. Folia Morphol. 2018; 77(2): 393-396, doi: 10.5603/FM.a2017.0084, indexed in Pubmed: 28933803.

6. Browning HC, Morton DE. The arterial pattern in the hand. Yale J Biol Med. 1955; 28(1): 37-55, indexed in Pubmed: 13257262.

7. Coleman SS, Anson BJ. Arterial patterns in the hand based upon a study of 650 specimens. Surg Ginecol Obstet. 1961; 113: 409-424, doi: 10.1097/00006534-19620100000028, indexed in Pubmed: 13694610.

8. Delorme E. De la ligature des artères de la paume de la main et en particulier des artères profondes et des artères de la plante du pied. Masson, Paris 1882.

9. Dujarier C. Anatomie des membres, dissection-anatomie topographique. G Steinheil, Paris 1905.

10. Earley M. The arterial supply of the thumb, first web and index finger and its surgical application. J Hand Surg Br. 1986; 11(2): 163-174, doi: 10.1016/0266-7681(86)90253-6, indexed in Pubmed: 3734551.

11. Erbil M, Aktekin M, Denk CC, et al. Arteries of the thumb originating from the superficial palmar arch: five cases. Surg Radiol Anat. 1999; 21(3): 217-220, doi: 10.1007/ BF01630906, indexed in Pubmed: 10431337.

12. Hashem AM, Knackstedt RW, Bernard S, et al. Variations in the origins and absence of the common digital arteries of the hand: a cadaveric study. J Hand Surg Eur Vol. 2018; 43(10): 1054-1058, doi: 10.1177/1753193418764289, indexed in Pubmed: 29558847.

13. Patil J, Kumar N, Aithal A, et al. An eccentric anatomical variation of palmar vascular pattern: Report of surgical challenging vascular variation. J Med Sci. 2016; 36(6): 240, doi: 10.4103/1011-4564.196372.

14. Singh S, Lazarus L, De Gama BZ, et al. An anatomical investigation of the superficial and deep palmar arches. Folia Morphol. 2017; 76(2): 219-225, doi: 10.5603/ FM.a2016.0050, indexed in Pubmed: 27665957.

15. Miletin J, Sukop A, Baca V, et al. Arterial supply of the thumb: systemic review. Clin Anat. 2017; 30(7): 963-973, doi: 10.1002/ca.22973, indexed in Pubmed: 28791730.

16. Murakami T, Takaya K, Outi H. The origin, course and distribution of arteries to the thumb, with special reference to the so-called A. princeps pollicis. Okajimas Folia Anat Jpn. 1969; 46(2): 123-137, doi: 10.2535/ofaj1936.46.23_123, indexed in Pubmed: 5820050.

17. Ottone N, Prum N, Dominguez M, et al. Analysis and clinical importance of superficial arterial palmar irrigation and its variants over 86 cases. Int J Morphol. 2010; 28(1), doi: 10.4067/s0717-95022010000100022.

18. Parks B, Arbelaez J, Horner R. Medical and surgical importance of the arterial blood supply of the thumb. J Hand Surgery Am. 1978; 3(4): 383-385, doi: 10.1016/s03635023(78)80044-6, indexed in Pubmed: 681725.

19. Ramírez AR, Gonzalez SM. Arteries of the thumb: description of anatomical variations and review of the literature. Plast Reconstr Surg. 2012; 129(3): 468e-476e, doi: 10.1097/PRS.0b013e3182402d43, indexed in Pubmed: 22373995.

20. Rodríguez-Niedenführ M, Burton GJ, Deu J, et al. Development of the arterial pattern in the upper limb of staged human embryos: normal development and anatomic variations. J Anat. 2001; 199(Pt 4): 407-417, doi: 10.1046/j.1469-7580.2001.19940407.x, indexed in Pubmed: 11693301.

21. Sappey CP. Trattato di Anattomia. Vol. 2. Detkin, Napoli 1897. 